\title{
Innovación y aplicación tecnológica en el ámbito de la Educación Superior universitaria. El empleo de los blogs en las universidades españolas.
}

\author{
Ma Carmen Salgado Santamaría \\ Universidad Complutense de Madrid \\ carmen.salgado@ccinf.ucm.es \\ Mª Julia GonzÁLEz CONDE \\ Universidad Complutense de Madrid \\ mariajul@ccinf.ucm.es \\ $\mathrm{M}^{\mathrm{a}}$ de las Mercedes Zamarra LópeZ \\ Universidad Complutense de Madrid \\ mzamarra@ccinf.ucm.es
}

\begin{abstract}
Resumen
La Universidad se encuentra inmersa en un escenario de cambio en el que las nuevas fórmulas docentes en tecnología y contenidos multimedia serán los principales aliados. Las tradicionales instituciones de educación superior, ya sean presenciales o a distancia, reajustan sus sistemas de distribución y comunicación. Pasan de ser el centro de la estrella de comunicación educativa a constituir simples nudos de un entramado de redes, dentro del gran ciberespacio, en donde el estudiante-usuario se mueve en unas coordenadas más flexibles. Del mismo modo, los cambios originados en estas nuevas coordenadas espacio-temporales traen consigo la aparición de distintas organizaciones de enseñanza, que se constituyen como consorcios o redes de instituciones y cuyos sistemas de enseñanza se caracterizan por la modularidad y la interconexión. Todo ello exige a las universidades una flexibilización en sus procedimientos habituales y en su estructura administrativa, para poder adaptarse a modalidades de formación alternativas más acordes con las necesidades que presenta esta nueva sociedad. Ahora, la implantación de plataformas blogs y otros recursos tecnológicos, contribuyen a la mejora de la calidad docente y, por consiguiente, a la formación y capacitación idónea del alumnado. Como una continuación de la innovación tecnológica, susceptible de ser adoptada en el ámbito universitario, surgen los blogs como un canal, herramienta o recurso de información y comunicación, cuya utilización se ha convertido en la revolución tecnológica que marca el inicio de la llamada Web 2.0.
\end{abstract}

Palabras clave: Blog, tecnología, universidad, innovación, docencia

Innovation and technological application in the field of higher education. The use of blogs in Spanish Universities.

\begin{abstract}
The University is immersed in a scenario of change in which new teaching formulas in technology and multimedia content will be the main allies. Traditional institutions of higher education, face-to-face or online, reset their distribution and communication systems. They go from being the center of educational communication star to form simple knots of a network of networks, inside the great cyberspace, where
\end{abstract}


the student/user is moving in a more flexible coordinate. In the same way, originated in these coordinates new space-temporal changes bring with them the emergence of different teaching organizations, which are consortia or networks of institutions and whose education systems are characterized by modularity and interconnection. All this requires universities easing in its usual procedures and its administrative structure, to be able to adapt to alternative forms of training more in line with the needs presented by this new partnership. Now, the implementation platforms blogs and other technological resources, contribute to the improvement of teaching quality and, therefore, to suitable training of students. As a continuation of the innovation technology, likely to be adopted at the University level, there are blogs as a channel, tool or resource of information and communication, whose use has become the technological revolution which marks the beginning of the so-called Web 2.0.

Key Words: Blog, Technology, University, Innovation, Teaching

\section{Referencia normalizada:}

Salgado Santamaría, C.; González Conde, M. J. y Zamarra López, M. M. (2013) Innovación y aplicación tecnológica en el ámbito de la Educación Superior universitaria. El empleo de los blogs en las universidades españolas. Historia y Comunicación Social. Vol. 18. N Especial Diciembre. Págs. 613-625.

Sumario: 1. Estado de la Cuestión. 2. Metodología. 3. Blogs y Universidad. 3.1 Funciones de los Blogs en el ámbito universitario. 3.2 Plataformas blogs en Universidades Españolas. 4. Conclusiones. 5. Referencias bibliográficas.

\section{Estado de la cuestión}

En estos momentos, la institución universitaria ávida de consolidación en la actualización y planteamiento de las nuevas técnicas y estructuras de comunicación y aprendizaje comienza a manifestarse más activa y flexible en cuanto a la adaptación social, desarrollando vías de integración de las tecnologías de la información y la comunicación en los procesos de formación. La irrupción de las nuevas tecnologías en todos los ámbitos cotidianos hace inevitable su uso en entornos educativos y exige una profunda reflexión en busca de una mejora en su potencial y en su adaptación a una actividad educativa tanto presencial como online. Estos grandes cambios en el ámbito de la educación nos hacen profundizar en la necesidad de renovación de la mayor parte de los fundamentos teóricos y prácticos que suponen la base existencial de una adecuada estrategia pedagógica.

Las universidades, se muestran cada vez más conscientes de los retos que supone dar una formación de calidad y apuestan por incorporar a sus entornos de aprendizaje, distintas herramientas tecnológicas para uso y beneficio, tanto, de sus cuadros docentes como de su población estudiantil. La incorporación de plataformas tecnológicas e-Learning vienen constituyéndose en un complemento cada vez mejor valorado por los docentes y estudiantes que ven en dichos mecanismos de encuentro e intercambio un método favorable para la publicación de apuntes, consignas de actuación, referencias documentales, casos de uso y tutoriales, prácticas, etcétera. 
De este modo, asegurando el paso hacia la innovación tecnológica, y a pesar de que el modelo didáctico ha variado levemente, y hasta en algunos casos sigue anclado en su sistema tradicional, basado en la presencia poco activa de los alumnos y en las clases magistrales, fundamentalmente teóricas, del profesor, lo cierto es que la manifestación de los nuevos recursos, las plataformas tecnológicas y el intercambio de experiencias y conocimientos de estos nuevos sistemas de comunicación y aprendizaje, abanderados por Internet, han dado un nuevo rumbo y otorgado un valor añadido a la docencia universitaria.

Distintos procedimientos tecnológicos innovadores aparecen y se expanden con fuerza por diversos entornos académicos y también en las aulas universitarias, hasta el punto de convertirse en elementos metodológicos imprescindibles que nos permiten hablar de un avance importante en sus criterios de modernización, aunque, no siempre en la proporción deseada. Sin embargo, en estos momentos cualquier universidad española, grande o pequeña, pública o privada, cuenta con un campus virtual, comparte blogs personales con institucionales y participa en wikis junto con otras tecnologías adyacentes, mejor o peor vistas, pero existentes, capaces de crear y desarrollar un gran número de contenidos virtuales que faciliten la labor de intercambio de enseñanza-aprendizaje tanto presencial como online.

Actualmente, la implantación de plataformas blogs y otros medios tecnológicos, contribuyen a la mejora de la calidad docente y, por consiguiente, a la formación y capacitación idónea del alumnado. El uso y aplicación de los blogs tanto por parte de las universidades como por la del profesorado, adscrito a diferentes centros, se viene expandiendo por la necesidad de conexión y adecuación a unos estudiantes que demandan más cercanía, más participación, más responsabilidad más proactividad y, en definitiva, más método experimental en la recepción e intercambio de conocimientos que se consideran sustanciales para su formación académica y profesional. En este sentido, los blogs, entre otros sistemas y plataformas tecnológicas, sirven de herramientas y recursos pedagógicos muy rentables para el corpus universitario. Su facilidad de uso, creatividad y originalidad en su manejo, su capacidad como dinamizador de actividades y adaptabilidad a todas las especializaciones y enseñanzas formativas, genera actitudes muy positivas entre los jóvenes lo que reporta beneficios realmente apreciables por su alto grado de rendimiento tecnológico y de conocimiento.

Los blogs, cuadernos de bitácoras o páginas web de fácil creación, se han constituido en un fenómeno imparable, cuya presencia se hace sentir en todos y a cada uno de los distintos ámbitos de conocimiento y estratos sociales, debido, precisamente, a su facilidad de uso y aplicación, alcanzando una magnitud y relevancia que no ha dejado ajenas a las universidades. Así, fueron las americanas las pioneras en incorporar comunidades de blogs entre sus docentes, investigadores y estudiantes universitarios. Y, en España, docentes e investigadores de diferentes universidades y comunidades autónomas, vienen creando, por iniciativa personal, sus propios blogs que van incorporando, de manera paulatina y experimental, como cuaderno de apuntes en línea y/o como herramienta de trabajo colaborativo. Sin embargo, del mismo modo, comprobamos que su presencia, adjunta a los logotipos de universidad, es 
muy reducida, a diferencia de cómo expresamente se manifiestan las universidades americanas. Inicialmente, sólo la Universitat Oberta de Catalunya y la Universidad de Granada, han mantenido un enfoque institucional de sus blogs, dando testimonio explícito de su utilización incipiente al inscribirlos, en ambos casos, junto con sus logotipos.

En cualquier caso, los promotores en el uso de estas plataformas tecnológicas, encuentran en los blogs una forma adecuada de comunicación e interacción entre profesor y alumnos. De este modo, un blog puede estar dedicado a una sola asignatura en la que el docente inserta avisos, publica documentación, proporciona enlaces de interés y permite que sus alumnos participen e interaccionen fuera de la universidad y de los horarios académicos. En este sentido, podemos afirmar que se produce una verdadera interactividad, un verdadero aprendizaje virtual sin ningún tipo de aplicación de costes millonarios. Valorando su aspecto práctico, los blogs pueden proporcionar diferentes posibilidades como ayuda en la relación profesor-alumno, apoyo a las clases presenciales, creación y desarrollo de contenidos virtuales, refuerzo tutorial $\mathrm{y}$ el resto de interacciones del extenso y complejo ámbito docente universitario.

Con el uso del blog como herramienta docente y comunicativa, el profesor no está sujeto y encorsetado por las herramientas que le ofrece su universidad, no tiene porqué tener conocimientos de diseño y desarrollo o programación web y puede interrelacionar la información que ofrece entre diferentes universidades, compartiendo su información con colegas de otras universidades, llevando a su verdadero sentido y fin último el concepto de transferencia de conocimiento.

\section{Metodología}

El objetivo de nuestra investigación es identificar las plataformas universitarias españolas que dan acceso y visibilidad virtual a los blogs integrados en su sistema educativo. Se trata de una muestra representativa, cuyo criterio de selección se basa sólo en aquellas Universidades que tienen un mayor número de estudiantes matriculados, en las distintas comunidades autónomas.

El método de análisis seguido en nuestra investigación ${ }^{1}$, consiste en la búsqueda pormenorizada en cada página web de las universidades elegidas para tratar de encontrar algún tipo de plataforma de blogs desde la que se oriente al usuario hacia los blogs relacionados con las actividades de la institución universitaria.

Dentro de las categorías de blogs hemos tenidos en cuenta tanto los oficiales o alentados por la propia institución, como los de carácter particular que a veces crean los propios alumnos o incluso los profesores. Las condiciones que deben cumplir para incluirlos en este informe son que estuviesen relacionados con las asignaturas y las enseñanzas que se imparten. 
Hemos deFIGGRno aceptar aquellos otros blogs de carácter personal o de otro tipo, aunque en cualquier caso, tampoco se excluirían los de carácter académico que pudieran hacer referencias de tipo personal, alguna mención a vacaciones, ocio, aficiones, etc. En un principio, la búsqueda de las plataformas se centró en las que fuesen de carácter oficial de las instituciones universitarias, pero a la vista de los pobres resultados iniciales que se obtenían, y que más adelante se detallarán, se amplió hasta las plataformas de carácter privado surgidas por la iniciativa particular, siempre que tuviesen relación con el ámbito académico.

\section{Blogs y Universidad}

Entre las estrategias que pueden ofrecer las universidades para animar y motivar a los profesores a entrar en el mundo de las tecnologías de aprendizaje están las de ofrecer un servicio de creación de blogs en sus servidores. Los blogs pueden ser herramientas que complementan los sistemas de impartición de cursos y en algunos casos pueden muy bien sustituirlos.

El hecho de que los blogs sean sencillos en su utilización, como hemos dicho en varias ocasiones, los convierte en herramientas ideales para las facultades o centros que no han tenido éxito en el uso de otros sistemas. Más aún, por ser herramientas que fomentan el acceso abierto a los contenidos, los blogs pueden desarrollar entre los académicos el espíritu de colaboración dentro de la universidad.

En términos prácticos, es relativamente sencillo implementar un proyecto de blogs dentro de una Universidad. En la mayor parte de los casos existe ya la infraestructura adecuada y lo que se necesita es espacio en un servidor de la propia universidad. Si se utiliza Blogger o WordPress, como sistemas gratuitos y de código abierto, lo que hará falta son los recursos humanos para formar a los usuarios y dar mantenimiento al sistema.

Es importante aclarar qué entendemos por blogs científicos, académicos o Science Blogs, como se denominan en el mundo anglosajón. Aunque todos los blogs tienen un soporte y una filosofía común, los bloggers suelen aglutinarse y organizarse en comunidades menores según los temas tratados, el origen geográfico o los intereses de sus autores. Un ejemplo evidente es la biblioblogosfera, biblogsfera o bibliogsfera, términos empleados para denominar los blogs del ámbito de la Documentación.

En principio, para establecer el marco al que se circunscriben los blogs científicos podrían emplearse al menos dos perspectivas. En primer lugar se podría asumir que todas aquellas bitácoras que hablan sobre temas relativos a la ciencia podrían considerarse científicas. Sin embargo, si seguimos esta perspectiva habría que incluir como mínimo los 24.793 blogs que el Directory Search de Technorati incluye bajo el paraguas del término Science (Frias, 2008. Pág. 775). 
En el caso español, su expansión en las universidades es escasa, aunque algunos docentes, vienen utilizando los blogs como iniciativa personal. Docentes e investigadores de diferentes universidades, por iniciativa propia están creando sus bitácoras e incorporándolas, bien, como un cuaderno de apuntes en línea o bien como herramienta de trabajo colaborativo.

\subsection{Funciones de los blogs en el ámbito universitario}

El empleo de blogs dentro del ámbito universitario puede estar orientado al cumplimiento de algunas de estas funciones:

Función institucional: Mantener viva la comunicación, el debate y la participación en una institución. Esta función es más fácil de desarrollar a nivel de centro o instituto que a nivel de toda la Universidad.

Función de acción social: Los blogs pueden servir de instrumento para el cambio social promovido desde la Universidad.

Función de promoción de Universidad y profesores: Profesores e investigadores pueden desarrollar sus propios blogs de forma independiente a la Universidad, pero su vinculación supone una promoción tanto para el profesor, como para la Universidad, que muestra, de este modo, los "talentos" con los que cuenta.

Función de promoción de los alumnos: Los blogs de alumnos, hechos bajo dirección académica, pueden centralizar su trabajo y progreso académico, sirviendo de carta de presentación para su incorporación al mundo profesional o académico.

Función de divulgación científica: Los blogs pueden llenar la brecha entre el mundo académico y la sociedad.

Función de transmisión del conocimiento científico: Los blogs pueden usarse como ágil mecanismo de revisión de trabajos científicos.

Siempre en línea con la innovación tecnológica, abundan de forma creciente los análisis, estudios e iniciativas que tienden a demostrar que el desarrollo de las habilidades de los alumnos en creatividad e innovación (dos variables claves en la formación actual y futura) se ve claramente favorecido por la utilización de recursos vinculados a la web 2.0. Por otra parte, desde la Universidad, existe un número creciente de profesores que son sensibles a utilizar esta nueva tendencia de los blogs y pretenden adaptarse a ella.

\subsection{Plataformas blogs en universidades españolas}

En este apartado mostramos los resultados obtenidos en cada una de las universidades, si bien, podemos sintetizar que la Universidad de Valencia es la que presta más atención al fenómeno de los blogs, aunque cuando lo que nos interesa es la localización de blogs tecnológicos, destaca la Autónoma de Madrid al incluir un instrumento dedicado a la localización de blogs especializados en tecnología $\mathrm{I}+\mathrm{D}+\mathrm{i}$. El fomento 
del uso de blogs ha sido potenciado por la Universidad de la Rioja a través de un concurso; y el blog que ha alcanzado casi el nivel de aula virtual, es el blog La Blogmática, de la Universidad Miguel Hernández, un foro de referencia para el estudio y análisis de casos penales.

\subsubsection{Análisis web universitarias}

Universidad de Barcelona: Además del Blog del Rector. http://paginadelrector. ub.edu/ ofrece una plataforma relacionada fundamentalmente con las bibliotecas de las distintas facultades de esta institución, http://www.bib.ub.edu./es/servicios/blogs.

Universidad Autónoma de Barcelona. (Blogs de la Universitat Autònoma de Barcelona): http://blogs.uab.cat/: Es una plataforma basada en Wordpress MU que permite a toda la comunidad de esta Universidad crear su propio blog, nos encontramos con contenidos muy diversos, desde los más personales (sobre cocina, por ejemplo) a otros más específicos, de historia o publicidad.

Desde los 14 blogs iniciales han llegado a 70, según figura en el listado de blogs actual. Constituye una de las experiencias pioneras en el uso de blogs a nivel institucional, en una universidad catalana. Permite conocer los programas de formación y ayudas, tanto tecnológicas como pedagógicas, podrían ser de gran interés para otras instituciones. Es importante destacar, cómo se anuncia en la normas de uso, que se aplica Licencia Creative Commons por defecto a todos los contenidos. Además: Wikis UAB. Por ejemplo, la del Grup d'Interès en Innovació Docent en Educació Superior, UABtube.

Universidad Politécnica de Cataluña http:/upc.es: Este caso también resulta llamativo, puesto que aunque la página donde reside la plataforma es la web de la propia Universidad (que recordemos es una politécnica), sin embargo la plataforma es particular: Racó de Blogs AAUPC (Rincón de blogs de la Asociación de Amigos de la Universidad Politécnica de Cataluña).

Universidad de Valencia http://blogs.uv.es/uv/blogs.html: Es sin duda la que está cuidando con más atención el fenómeno de los blogs. En su plataforma Blogs de la Universitat de Valencia resulta muy sencillo moverse. Se define a sí misma como "la pretensión de ser el sistema oficial de blogs de la Universidad de Valencia". Además, en la página principal de la web se anuncia la creación de "un gran dispositivo digital para la enseñanza, la investigación y la cultura" llamado RODERIC (Recursos Oberts Digitals per a l'Ensenyament, la Recerca i la Cultura), en español significa "Rodrigo" y está dedicado al Papa Alejandro VI (Roderic de Borja), fundador de la Universidad de Valencia.

Además de esta plataforma oficial, esta Universidad cuenta con Red Bloguera U.V. en la que un grupo de personas relacionadas con esta Universidad, entre otras algunos alumnos, se han propuesto completar las lagunas que, según ellos, tiene la plataforma oficial. Desde el Servicio de Medios e Innovación Docente, la Universidad de Valencia intenta destacar la extensión y visibilidad social de la labor universitaria. En este 
caso, la Universidad de Valencia con Wordpress crea el sistema y las condiciones para que su uso sea extenso, crítico, innovador pedagógicamente hablando, socialmente influyente (la Universidad Politécnica de Madrid y la Autónoma de Barcelona ya lo habían hecho con anterioridad).

Universidad Complutense de Madrid. www.ucm.es: El Blog del Rector está definido como un espacio para la defensa de la Universidad Pública para impulsar los debates que afectan al mundo universitario que tiene su reflejo en la sociedad. Con anterioridad y a través de la Fundación General de la UCM comprometida con la mejora de la transmisión del conocimiento, la investigación y la formación humana integral se ofrece a los alumnos la opción de participar en la elaboración de un blog para conseguir créditos de libre configuración participando en grupos de trabajo con una materia en común. En esta dirección los alumnos interesados pueden ver un ejemplo del curso http://www.ucm.es/info/fgu/ciclos/tecnologia/cursos/mujer_ciencia.htm\#acceso. Por defecto se exige un mínimo de intervenciones para que los alumnos participen, si bien la verdadera intención de el blog es que los estudiantes comenten las noticias, eventos, se relacionen entre ellos, y participen en el foro de una manera dinámica.

También emplean esta nueva herramienta en la red las asociaciones de estudiantes, entre las que cabe destacar, por ejemplo, el blog de la Asociación de Jóvenes Investigadores Filólogos de la Universidad Complutense de Madrid: http://ajif-ucm.blogspot.com/. En esta bitácora los fundadores de la asociación explican la importancia del blog, las pautas a seguir por sus miembros, y sus cometidos. El blog ha sido en este caso un instrumento informativo previo a la creación de la página web oficial de la Asociación. El blog es fundamental para la difusión en la red de los acontecimientos de relevancia para la Asociación, para fomentar la investigación y dedicación filológica desde los primeros cursos hasta la lectura de tesis. Por otro lado, en la UCM también hay profesores que crean blogs relacionados con los proyectos o líneas de investigación.

Universidad Autónoma de Madrid http://www.uam.es/otros/cuturam/blogs.html: Esta plataforma se denomina Weblogs en Madri $+d$. Se trata de un instrumento dedicado a la localización de blogs especializados en tecnología $\mathrm{I}+\mathrm{D}+\mathrm{i}$ como se puede observar en el juego que realiza con la palabra Madrid: Madri+d.

Universidad Antonio de Nebrija http://blogs.nebrija.es: Esta Universidad privada cuenta con una plataforma de blogs, que contiene pocos blogs y algunos están todavía vacíos. Tiene el defecto, común a todas las demás estudiadas, de que por el momento no aparece ningún enlace en la página principal.

UNED http://persazul.blogspot.com: Parece tratarse de un blog de un alumno de la Universidad Nacional de Educación a Distancia en el que se ofrecen de forma particular enlaces, apuntes, fechas de exámenes, foros, etc. Contiene publicidad.

Universidad de La Rioja: La Universidad de La Rioja, el Instituto Riojano de la Juventud y www.larioja.com, ofrecen a los universitarios riojanos la oportunidad de 
demostrar todo su talento, en un concurso. Para ello tan sólo tendrán que abrir su propio blog. El ganador recibirá como premio un ordenador portátil y la satisfacción de que miles de internautas conozcan su trabajo. Las webs universitarias fueron objeto de un análisis en profundidad en los inicios de Universia, con el objetivo de alcanzar el mayor grado de complementariedad y apoyo en el diseño de un portal que debía acoger y dar servicios a centenares de universidades de un gran número de países. Universia ha puesto el primer blog universitario con la pretensión de unir en una comunidad a más de 12 millones de estudiantes de habla hispana y portuguesa. Este paso es el más ambicioso y puede que hasta revolucionario propiciado por Internet. Destaca por organizar un concurso a través de su blogs, denominado "Concurso 'Blog\&Roll in Rioja' para universitarios" y lo organizaron UR, IRJ y el portal larioja. com. El Concurso 'Blog\&Roll' fue una iniciativa de la Universidad de La Rioja, el Instituto Riojano de la Juventud y larioja.com dirigida a los estudiantes universitarios para fomentar el uso de esta herramienta de Internet. El certamen estuvo abierto tanto a los estudiantes de la Universidad de La Rioja como a los alumnos riojanos matriculados en otros campus. El único requisito era abrir un blog en el portal larioja. com, incluido en la sección Blog'n'roll. Los blogs abiertos antes del 15 de febrero no entrarían en el concurso, aunque los propietarios de blogs ya existentes tenían la posibilidad de abrir uno nuevo y participar. El jurado valoró aspectos como la calidad y presentación de los contenidos, originalidad y tratamiento del tema, inclusión de elementos multimedia, frecuencia de actualización, empleo de los recursos que permite el medio. (Enlaces a otras páginas, imágenes u otros elementos, recursos tipográficos, otros), la interacción con los visitantes del blog y la capacidad de atraer tráfico (visitantes) al blog.

En la web larioja.com aparecía la opción de "Crea tu propio blog", vincularlo a la categoría Blog\&Roll. El ganador de este primer premio fue el alumno de Ingeniería Técnica en Informática de Gestión José Antonio Lázaro Espila, por su bitácora Mi madre no estará orgullosa en la que creó 142 entradas y recibió 1.140 comentarios.

Universidad Miguel Hernández: Más allá de los blogs de profesores dedicados a diverso temas medioambientales y de salud, destaca La Blogmática Penal: el Derecho penal en La Red, es un blog dedicado a la docencia, investigación y práctica del Derecho penal de dicha Universidad http://blogmaticapenal.blogspot.com.es. $L a$ Blogmática es una iniciativa docente que pretende aprovechar todo el tiempo que pasan en la red, para el aprendizaje en los conocimientos y habilidades relacionados con la práctica del Derecho penal. Con un diseño moderno e innovador, el blog es obra del profesor de Derecho penal de la UMH, Fernando Miró Llinares y de los alumnos de la licenciatura en Derecho: Lisardo Vidal Marín y Jesús Gil Morales. La Blogmática en una herramienta útil para que los estudiantes discutan la solución a casos judiciales, conozcan referencias bibliográficas imprescindibles o comenten sus dudas con los profesores del área; pero también pretende convertirse en una cafetería virtual en la que profesores de otras universidades cuenten sus experiencias y otras perspectivas en la docencia del Derecho penal. La blogmática cuenta con una página principal en la que se informan de las diferentes actualizaciones semanales, 
comentando otras cuestiones generales, y con 5 secciones: historias de pasillo, lecturas recomendadas, el caso, el cajón del penalista, noticias y el foro. En la sección historias de pasillo cuelgan anécdotas, experiencias y otras historias de catedráticos y profesores titulares de Derecho penal de otras universidades, magistrados, fiscales o abogados dedicados a la práctica del Derecho penal e incluso de estudiantes Erasmus de otras Universidades o de becarios de investigación. De esta forma, la blogmática se convierte en un aula práctica virtual en la que se debatirán los casos de más actualidad.

Universidad de Granada: Es pionera en la creación de un Blog Institucional creado por el Vicerrectorado de Alumnos en junio de 2006. Un espacio común para los estudiantes en un campus español. El blog va destinado principalmente a los estudiantes universitarios en el cual podrán encontrar la información que les interesa y verter sus propias ideas y experiencias. Este blog tiene como finalidad contar de una manera sencilla, clara y cercana la actualidad de lo que acontece en la Universidad, además de convertirse en un espacio en el que los universitarios se encuentren cómodos para exponer sus opiniones sobre temas académicos, escuchar las opiniones de otros de sus compañeros, abrir coloquios, exponer quejas. Además en la página se pueden leer apartados fijos como el de "Cartas al Vicerrectorado de Estudiantes (VE)", "Entrevista al Personal del VE", entre otros. Pero no sólo se puede escribir sobre temas estrictamente académicos, sino también críticas sobre las últimas lecturas, cosas interesantes que hayas encontrado en la web o recomendaciones de otros blogs igualmente interesantes.

Universidad de Sevilla: La Facultad de Comunicación de la Universidad de Sevilla ha desarrollado Blogosfera fcom, bajo el tema e-learning, conocimiento en red y web colectiva, en el que desarrollan los siguientes puntos:

Fcom para el usuario: Proyecto de desarrollo colectivo de la nueva web.

escenarte//cultura: Blog enciclopedia de las artes contemporáneas fruto del trabajo en conjunto del SMID y alumnos de la asignatura de Movimientos Artísticos Contemporáneos del profesor Antonio Molina, vicedecano de Innovación Docente de la Facultad.

Manuel Ángel Vázquez Medel: Blog sobre Estudios Culturales, Creatividad y Comunicación, el catedrático de literatura Vazquez Medel. Servicio de Medios e Innovación Docente -El blog del SMID-.

Innovación Docente: Observatorio de Innovación Educativa.

Empresas de Comunicación: Un blog de Francisco Caro. Profesor del Departamento de Administración de Empresas y Comercialización e Investigación de Mercados.

wiki escenarte: Enciclopedia de las artes contemporáneas. 
Universidad de Las Palmas www.ulpgc.es: Desde la página principal, a través de un buscador general, se puede acceder a un servicio de blogs del Departamento de Informática y Sistemas, pero hay que registrarse.

Universidad de Jaén, ujaen.es.sci/redes/web/blogs/index.htm: Esta Universidad tiene blog, al que se accede a través del buscador de la página principal. Hay que darse de alta en el Blog Institucional o en el Blog que requiere su activación en el Campus Virtual.

\section{Conclusiones}

En estos momentos, sólo un reducido número de universidades españolas utilizan los blogs de manera pública y explícita para todos sus usuarios.

Los docentes universitarios españoles empiezan a utilizar los blogs, de manera incipiente y paulatina, en la mayor parte por iniciativa propia y muchas veces sin el aval institucional en donde desarrollan su labor docente.

Los blogs no pueden ser considerados como elementos estáticos sino en continua evolución para servir como una de las herramientas estratégicas de primer orden -junto con los campus virtuales-, para alcanzar los distintos compromisos insertados en el Espacio Europeo de Enseñanza Superior.

La presencia de estos blogs, adscritos a la actividad universitaria, debe estar explícita y visiblemente expuesta en su página web para su identificación, acceso y muestra de calidad y renovación docente.

Dentro de nuestras conclusiones sectoriales, reseñamos una pequeña muestra de aquellas universidades españolas que han sido pioneras en alguna gestión de innovación en el uso y aplicación de sus blogs:

La Universidad de Valencia es la que muestra mayor interés por el fenómeno de los blogs

El blog de la Universidad Miguel Hernández, La Blogmática es un foro de referencia para el estudio y análisis de casos penales.

La Universidad de Granada es precursora en la creación de un Blog Institucional.

La Universidad Complutense de Madrid ofrece reconocimiento de créditos por la participación en Blogs.

Es importante que la Universidad española, en su conjunto, readapte, acentúe, institucionalice y consolide las nuevas metodologías y los nuevos procesos de enseñanza basados en la innovación tecnológica, entre los que se define el blog como una de las herramientas que proporciona un gran rendimiento académico, bajo unas condiciones accesibles, asequibles y fáciles de manejar por toda la comunidad universitaria. 


\section{Referencias bibliográficas}

AGUADED GÓMEZ, I (2005). "Estrategias de edu-comunicación en la sociedad audiovisual". En Comunicar, n' 24.

CALVO, E.; MARTÍNEZ, S.; ZAMARRA, M (2010). "Pautas para la construcción de un protocolo de interrelación entre blogs y campus virtuales". En La Comunicación Social, en estado crítico. Entre el mercado y la comunicación para la libertad. La Laguna, Tenerife: Sociedad Latina de Comunicación Social.

CASTELLS, M. (2001). La galaxia Internet. Madrid: Editorial Plaza \& Janés.

CEBRIAN, M.; ESTEVE, F. (2008): Blogalaxia y periodismo en la red: Estudio, análisis y reflexiones. Madrid: Editorial Fragua.

CEREZO, J. M. (2006). Blogosfera Hispana: pioneros de la cultura digital. Fundación France Tecom España.

FLORES VIVAR, J.M. (et al.) (2008). CIBER: (Blogs) Recursos de valor añadido para la optimización de la docencia en el Campus Virtual. Madrid: Vicerrectorado de Desarrollo y Calidad de la Docencia. Servicio de Publicaciones Universidad Complutense de Madrid.

FOMBONA, J.; PASCUAL, M.A. (2013). Audiovisual resources in formal and informal learning: Spanish and Mexican students' attitudes. International Education Studies Vol. 6, nº 2, February 2013, p.1-11.

FRÍAS MONTOYA, J. M.; TRAVIESO RODRÍGUEZ, C. (Eds.). (2008): Formación, investigación y mercado laboral en Información y Documentación en España y Portugal. Ediciones Universidad de Salamanca.

GONZÁLEZ CONDE, M.J. (2009) "Interactividad a toda costa”, en Flores Vivar y Esteve Ramírez, F. (Eds.) Periodismo Web 2.0. Madrid: Fragua. p. 341-347.

GONZÁLEZ, M.J.; SALGADO, C.; FOMBONA J. (2012). "Competencias didácticas en cursos on line de posgrado universitario". En Estudios sobre el Mensaje Periodístico. Vol. 18. Madrid. p. 443-452.

KISS, D. y Castro, E. (2005). Interacción comunicativa con la tecnología informática, en Educación en Comunicación. Hacia un curriculum iberoamericano. En Comunicar, $\mathrm{n}^{\circ} 24$.

PADILLA, G; SALGADO, C.; COBO, F. (2011). El reto del EEES en docencia e investigación en el área de los medios sociales. Madrid: Visión Libros.

PÉREZ TORNERO, J.M. (2000). Las escuelas de enseñanza en la sociedad de la información, en Pérez Tornero (comp.): Comunicación y educación en la sociedad de la información. Barcelona: Paidós.

ROJAS, O. (2005): Blogs. Madrid: ESIC.

RUBIO JORDÁN, A.V. (2012): "El uso de plataformas sincrónicas aplicadas a la enseñanza del periodismo". En Revista de Comunicación Vivat Academia n¹17, febrero 2012. Madrid. UCM.

RUIZ-MORA, I.; OLMEDO-SALAR, S.: "Desarrollo de competencias profesionales en el Espacio europeo de educación superior (EEES). Nuevas Metodologías en los estudios de comunicación"." En Revista de Comunicación Vivat Academia $\mathrm{n}^{\circ} 117$, febrero 2012. Madrid. UCM. 
SALAVERRÍA, R. (2005). Cibermedios. El impacto de internet en los medios de comunicación en España. Sevilla: Comunicación Social.

SALGADO, C. (2012). Sistemas docentes en el EEES. Madrid: Editorial Visión Libros.

SALGADO, C. (2012). "El EEES un destino seguro a distintas velocidades", en El reto de la innovación docente. El EEES como punto de encuentro. Madrid: Editorial Visión Libros.

SALMI, J. (2009). El desafio de crear universidades de rango mundial. Banco Internacional de Reconstrucción y Fomento/Banco Mundial, coedición con Mayol Ediciones S.A. Colombia.

SILVA DE DEL VALLE, R. (2012): "Evaluación de un módulo formativo del programa universitario de formación integral: Prometeo" En Revista de Comunicación SEECI n²9, noviembre 2012. Madrid: UCM.

PARRA, D. (2008). "De Internet 0 a Web 3.0: un reto epistemológico para la comunidad universitaria". En Anàlisi, n ${ }^{\circ}$ 36, Barcelona.

TAPSCOTT, D.; WILLIAMS, A. (2008). Wikinomics. Barcelona: Editorial Paidós Empresa.

ZAMARRA LÓPEZ, M.M. (et al.) (2007). Innovación y métodos de aprendizaje en el EEES. Madrid: Facultad de Ciencias de la Información (UCM).

ZAMARRA LÓPEZ, M.M. (et al.) (2007). WYRIWYL: Campus Virtual, Internet y otras tecnologías emergentes y su aplicación metodológica en los nuevos escenarios de enseñanza-aprendizaje. Madrid: Vicerrectorado de Innovación y Espacio Europeo de Educación Superior (UCM).

ZAMARRA LÓPEZ, M.M. (2002): "El periodismo digital y la nueva formación ciberperiodística". En Revista de Comunicación SEECI nº, noviembre 2002. Madrid: UCM.

\section{Notas}

1 CIBER: Proyectos de Innovación y Mejora de la Calidad Docente UCM (Referencia Nro.76). Proyecto de innovación y mejora de la calidad docente UCM 173/2013 "Diseño y desarrollo de AVA con contenido que permitan la conceptualización y apropiación de medios tecnológicos con énfasis en el trabajo colaborativo y herramientas TIC. 